\title{
Cumulative luminosity functions of the X-ray point source population in M31
}

\author{
L. Shaw Greening, ${ }^{1}$ C. Tonkin,,${ }^{1}$ R. Barnard,${ }^{1}$ U. Kolb ${ }^{1}$ \\ and J. P. Osborne ${ }^{2}$ \\ ${ }^{1}$ Open University, Walton Hall, Milton Keynes, MK7 6AA, UK \\ email: L.Shaw-Greening@open.ac.uk \\ ${ }^{2}$ University of Leicester, University Road, Leicester, LE1 7RH, UK
}

\section{Introduction}

We present preliminary results from a detailed analysis of the X-ray point sources in the XMM-Newton survey of M31 (e.g. Barnard et al. 2005; Pietsch et al. 2005). These sources are expected to be mostly X-ray binaries.

\section{Source Detection}

The left hand panel of Fig. 1 shows a mosaic of GALEX FUV images of M31 (Thilker et al. 2005) overlaid by the point sources detected by edetect_chain in SAS 6.0.0. Only sources which were on both the PN and MOS chips were analysed. The minimum detection likelihood was set to 10 and detections that overlapped at a radius of $20^{\prime \prime}$ were excluded. Lightcurves, energy spectra and power density spectra were then extracted for each region and analysed to determine source properties. Energy spectra were fit with power law, bremsstrahlung, black body and neutron star atmosphere models in xspec 11.3.1 with free parameters to determine the flux of the source. The unabsorbed luminosities were then calculated assuming a distance to M31 of $760 \mathrm{kpc}$ (van den Bergh 2000).

\section{Results}

In the right hand panel of Fig. 1, we present the first cumulative luminosity functions of the XMM-Newton M31 fields to be created using individual fits for each source spectrum to obtain the unabsorbed luminosity for each source. The North 1 (solid) and 2 (dashed) field luminosity functions are very similar in shape despite having very different numbers of sources. The Core and South 1 luminosity functions have different shapes because these fields have not yet been fully surveyed down to the same luminosity limits as the North fields. The final luminosity limit for each field will also depend on the good-time interval of each observation. We will investigate the significance of these luminosity functions once the survey is complete.

We have also created a luminosity function of the North 1 and 2 fields combined in order to compare it to the luminosity function of these fields reported by Trudolyubov et al. (2002). Unlike in our study, these authors used individual spectral fits only for the brightest sources $\left(L_{X}>5 \times 10^{36} \mathrm{erg} \mathrm{s}^{-1}\right)$; the faint sources had their luminosities estimated assuming an absorbed power law model with fixed power law index. Above $\mathrm{L}_{X}=10^{36} \mathrm{erg} \mathrm{s}^{-1}$ our luminosity function can be approximated by a power law $N(L>$ $\left.L_{X}\right) \sim L_{X}^{-\alpha}$ with slope $\alpha=0.87$, inconsistent with $\alpha=1.3$ found by Trudolyubov et al. (2002). This is probably a consequence of the fixed spectral model assumed by these authors.

Kong et al. (2002) used Chandra to study the core region of M31. They extracted energy spectra from the longest $(8.8 \mathrm{ks})$ observation and carried out spectral fitting only 

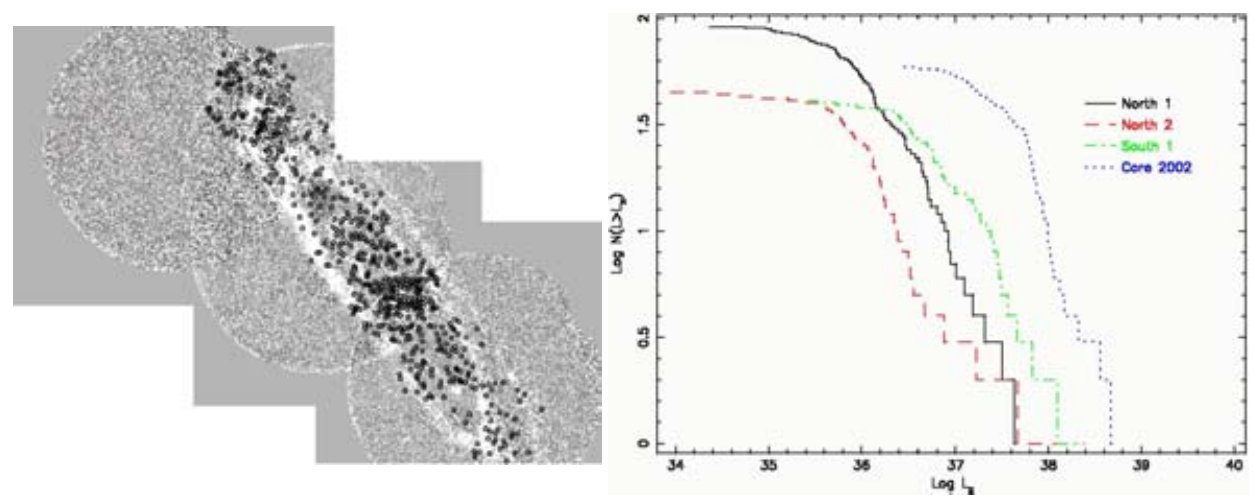

Figure 1. The left hand panel shows the X-ray sources detected, superimposed on a mosaic of GALEX FUV observations of M31. The right hand panel shows the luminosity functions of each of the fields we have studied.

on the brightest 20 of the 204 sources they found. All sources were well fit by both absorbed power law and Raymond-Smith models. For the other 184 sources, count rates were converted to luminosities assuming an absorbed power law model. They obtained a luminosity function described by $\alpha=1.44$ above a break at $1.77 \times 10^{37} \mathrm{erg} \mathrm{s}^{-1}$ for the whole core exposure. This is comparable with our $\alpha=1.96$ for $37.7<\log \left(\mathrm{L}_{X}\right.$ erg $\left.\mathrm{s}^{-1}\right)<38.2$ in the Core luminosity function.

Grimm et al. (2002) studied the Milky Way and created luminosity functions for the high and low mass binary populations separately. Their cumulative luminosity functions had $\alpha=0.64$ for HMXBs and $\alpha=0.26$ for LMXBs. Neither of these slopes is consistent with our luminosity functions of M31 but the binary population cannot be easily separated in M31 and both types of binary are present.

\section{Future Work}

We have so far studied 225 sources over 4 fields and our survey is continuing by looking at the unstudied sources we have detected in the 2 outermost disc fields, the 130 fainter core sources and the 51 fainter sources in the South 1 field. X-ray binaries will be identified by their energy spectrum and power density spectrum as outlined in Barnard et al. $(2004,2005)$. We will also continue to create luminosity functions for each field to allow us to compare populations. All the luminosity functions we create will also need to be corrected for foreground and background sources (Giacconi et al. 2001; Hasinger et al. 2001) and for incompleteness (see Kim \& Fabbiano (2004))

\section{References}

Barnard, R., Kolb, U. \& Osborne, J. P. 2004, A\&A, 423, 147

-. 2005, astro-ph/0508284

Grimm, H.-J., Gilfanov, M. \& Sunyaev, R. 2002, A\&A, 391, 923

Giacconi, R., Rosati, P., Tozzi, P., et al. 2001, ApJ, 551, 624

Hasinger, G., Altieri, B., Arnaud, M., et al. 2001, A\&A, 365, L45

Kim, D.-W. \& Fabbiano, G. 2004, ApJ, 611, 846

Kong, A. K. H., Garcia, M. R., Primini, F. A., et al. 2002, ApJ, 577, 738

Pietsch, W., Freyberg, M. \& Haberl, F. 2005 A\&A 434, 483

Thilker, D. A., Hoopes, C. G., Bianchi, L., et al. 2005, ApJL, 619, L67

Trudolyubov, S. P., Borozdin, K. N., Priedhorsky, W. C., Mason, K. O. \& Cordova, F. A. 2002, ApJL, 571, L17

van den Bergh, S. 2000, PASP, 112, 529 